\title{
The impact of genomics evolution and genomic island in Burkholderia pseudomallei
}

\section{Opinion}

Burkholderia pseudomalleus is a Gram-negative, bipolar, aerobic, motile rod-shaped bacterium. ${ }^{1}$ It is a soil-dwelling bacterium endemic in tropical and subtropical regions worldwide, particularly in Thailand and northern Australia. ${ }^{2}$ It's a powerful organism, can live in different environments is also capable to cause a potentially life-threatening infection. It infects humans and animals and causes the disease melioidosis, there to be 165,000 (95\% credible interval 68,000 412,000 ) human melioidosis cases per year worldwide, of which $89,000(36,000-227,000)$ die. The mortality rates of Melioidosis are often affected by the difficulty to diagnose the disease due to its large range of symptoms. ${ }^{3}$ It is also capable of infecting plants. ${ }^{4}$ B. pseudomallei are an opportunistic pathogen. An environmental organism, it has no requirement to pass through an animal host to replicate.

$B$.pseudomalleus is capable of infecting almost anything because of the spectacular genetic structure, by possessing certain genomic island (GIs) that gives the ability to alteration and to adapt. The genes of this GIs play an important role in pathogenesis ability. Trains which cause disease in humans differ from those causing disease in other animals, Genes on these genomic islands may thus play an important role in the pathogenesis of human melioidosis. It may have the ability to cause disease in humans because of DNA it has acquired from other microorganisms. ${ }^{5}$ Its mutation rate is also high, and the organism continues to evolve even after infecting a host. ${ }^{6}$ These genetic alterations were primarily driven by environmental pressures outside the human or mammalian host.?

The bacteria undergo for different changes to survivor in all kind of condition, the genomes evolve through mutations, rearrangements or horizontal gene transfer that contributes to the diversification and adaptation of microorganisms which is or has been facilitated by genomic islands (GEIs). Besides the core genes encoding essential metabolic functions, bacterial genomes also harbour a number of accessory genes acquired by horizontal gene transfer that might be beneficial under certain environmental conditions, thus having an impact on the genome plasticity. GEIs are discrete DNA segments, some of which are mobile and others which are not, or are no longer mobile, which differ among closely related strains. That not all, is also characterized by genetic traits which is a number of GEIs are capable of integration into the chromosome of the host, excision, and transfer to a new host by transformation, conjugation or transduction. The ability of freely exchange genes feature contribute to dissemination of variable genes, including antibiotic resistance and virulence genes leading to generation of hospital 'superbugs', as well as catabolic genes leading to formation of new metabolic pathways. Both core gene and genomic island enhance bacteria survival opportunity environmental and pathogenic. ${ }^{8}$ Example; melioidosis disease which caused by Burkholderia pseudomalleus (BP) commonly presenting as an acute septicemic illness, chronic Bp infection is also well recognized which can be confused with TB or malignancy, this confusing happened because of the gene exchange. ${ }^{9}$
Volume 6 Issue 4 - 2017

\author{
Alaa A Elnour \\ Medical Laboratory Scientist, National Ribat University, Saudi \\ Arabia
}

Correspondence: Alaa A Elnour, Medical Laboratory Scientist, Ibrahim Malik Teaching Hospital National Ribat University, Saudi Arabia,Email alaa.a.elnour@outlook.com

Received: October 27, 2017 | Published: November 16, 2017

Many bacterial species exploit specialized secretion systems to transfer macromolecules across bacterial membranes, which by transfer of proteins or nucleoprotein complexes directly mediate pathogenicity and horizontal gene transfer. ${ }^{10}$

B. pseudomalleus has a highly plastic genome and has the ability to acquire new genes horizontally from other microorganisms, especially as the pathogen persists in the environment. A large-scale blast score ratio (LS-BSR).

GIs is one of the major sources of genomic diversity within $B$. pseudomallei and the molecular mechanisms that facilitate horizontally-acquired GIs are common across multiple strains of B. pseudomallei. The differential presence of the 71 GIs across multiple strains demonstrates the importance of these mobile elements for shaping the genetic composition of individual strains and populations within this bacterial species..$^{10}$ The genomic positions of these GIs are not random, as many of them are associated with tRNA gene loci. In particular, the $3^{\prime}$ end sequences of tRNA genes are predicted to be involved in the integration of GIs. ${ }^{11}$ B. pseudomallei genes that may cause a predisposition to human disease and that these genes tend to be located on GIs. ${ }^{12}$

B. pseudomallei, is composed of two chromosomes of $4.07 \mathrm{megabase}$ pairs and $3.17 \mathrm{megabase}$ pairs, each one of them genes have various function. The large chromosome encodes many of the core functions associated with central metabolism and cell growth, whereas the small chromosome carries more accessory functions associated with adaptation and survival in different niches. Genomic comparisons with closely and more distantly related bacteria revealed a greater level of gene order conservation and a greater number of orthologous genes on the large chromosome, suggesting that the two relicense have distinct evolutionary origins. A striking feature of the genome was the presence of 16 genomic islands (GIs) that together made up $6.1 \%$ of the genome, ${ }^{13}$ Unfortunately, almost $35 \%$ of the GI genes encode 'hypothetical' proteins, meaning that their function is unknown. ${ }^{12}$

B. pseudomallei genome is widely considered to be 'open' (sequencing new strains is always expected to lead to the discovery of new genes), with very high levels of lateral gene transfer, generating the large and moderately diverse genome observed, that recombines at a high frequency, leading to great intra-species diversity within and 
among pathogen populations. Demonstrated high levels of diversity in B. pseudomallei in datasets from MLST (Multi-Locus Sequence Typing) analysis suggesting that genetic exchange has not been limited to GIs. These conserved MLST sites are located at scattered locations along the genome and suggest that homologous exchange occurs extensively throughout the genome and includes presumably core 'housekeeping' genes. ${ }^{14}$

We can use pathogenicity islands (PAIs) as marker to help identify a certain bacterial pathogen, PALs are a distinct class of genomic islands (GEIs), which are acquired by horizontal gene transfer. PAIs harbour virulence genes and some, in addition, antibiotic resistance genes. More often genes conferring antibiotic resistance are encoded by GEIs not containing virulence genes. Both types of genetic elements are found in genomes of various human, animal and plant pathogens. There are PAIs and GEIs which are specific for a certain serotype(s), strain, or pathotype of a species. Furthermore, there are also PAIs which are more widespread and found in bacterial pathogens causing a certain pathogenic effect in the host. Even the lack of a certain PAI might be characteristic for a defined subspecies. Obviously, PAIs can be used as markers for diagnostic purposes to help identify a certain bacterial pathogen, subtype it, estimate the pathogenic potential, and in some cases predict its antibiotic resistance. ${ }^{15}$

The genomic structure of bacteria, gives them the ability to survive in new environment and respond to environmental stress and also strengthen the pathogenic effect, so small organism with high life threatening danger.

\section{Acknowledgements}

None.

\section{Conflict of interest}

The author declares no conflict of interest.

\section{References}

1. Virginia Tech Pathogen Database. Archived from the original on 200609-01.

2. Limmathurotsakul D, Golding N, Dance DA, et al. Predicted global distribution of Burkholderia pseudomallei and burden of melioidosis. Nat Microbiol. 2016;1(1):15008.
3. Melioidosis, pseudoglanders Whitemore's Disease. USA: Iowa State University, The Center for Food Security and Public Health; 2016.

4. Lee YH, Chen Y, Ouyang X, et al. Identification of tomato plant as a novel host model for Burkholderia pseudomallei. BMC Microbiol. 2010;29:10-28.

5. Sim $\mathrm{SH}, \mathrm{Yu} \mathrm{Y}$, Lin $\mathrm{CH}$, et al. The core and accessory genomes of Burkholderia pseudomallei: implications for human melioidosis. PLoS Pathog. 2008;4(10):e1000178.

6. Price EP, Hornstra HM, Limmathurotsakul D, et al. Within-host evolution of Burkholderia pseudomallei in four cases of acute melioidosis. PLOS Pathog. 2010;6(1):e1000725.

7. Nandi T, Ong C, Singh AP, et al. A Genomic Survey of Positive Selection in Burkholderia pseudomallei Provides Insights into the Evolution of Accidental Virulence. PLoS Pathog. 2010;6(4):e1000845.

8. Tuanyok A1, Leadem BR, Auerbach RK, et al. Genomic islands from five strains of Burkholderia pseudomallei. BMC Genomics. 2008;9:566.

9. Juhas M, van der Meer JR, Gaillard M, et al. Genomic islands: tools of bacterial horizontal gene transfer and evolution. FEMS Microbiol Rev. 2008;33(2):376-393.

10. Sarovich DS, Price EP, Webb JR, et al. Variable Virulence Factors in Burkholderia pseudomallei (Melioidosis) Associated with Human Disease. PLoS One. 2014;9(3):e91682.

11. Tuanyok A, Leadem BR, Auerbach RK, et al. Genomic islands from five strains of Burkholderia pseudomallei. BMC Genomics. 2008;9:566.

12. Sim SH, Yu Y, Lin $\mathrm{CH}$, et al. The Core and Accessory Genomes of Burkholderia pseudomallei: Implications for Human Melioidosis. PLoS Pathog. 2008;4(10):e1000178.

13. Matthew TG Holden, Titball RW, Peacock SJ, et al. Genomic plasticity of the causative agent of melioidosis, Burkholderia pseudomallei. In: Peter Greenberg E, editor. USA: University of Iowa; 2004. p. 1-6.

14. Spring-Pearson SM, Stone JK, Doyle A, et al. Pangenome Analysis of Burkholderia pseudomallei: Genome Evolution Preserves Gene Order despite High Recombination Rates. PLoS One. 2015;10(10):e0140274.

15. Oelschlaeger TA, Hacker J. Impact of pathogenicity islands in bacterial diagnostics. APMIS. 2004;112(11-12):930-936. 